\title{
Genotyping of 353 Staphylococcus aureus Bloodstream Isolates Collected between 2004 and 2009 at a Norwegian University Hospital and Potential Associations with Clinical Parameters
}

\author{
Hege Vangstein Aamot, ${ }^{a}$ Anita Blomfeldt, ${ }^{a, b}$ and Arne N. Eskesen ${ }^{c}$ \\ Department of Microbiology and Infection Control, Division of Diagnostics and Technology, Akershus University Hospital, Lørenskog, Norwaya; Department of Clinical \\ Molecular Biology and Laboratory Sciences (EpiGen), Division of Medicine, Akershus University Hospital, University of Oslo, Oslo, Norway ${ }^{\mathrm{b}}$; and Department of Infectious \\ Diseases, Division of Medicine, Akershus University Hospital, Lørenskog, Norwayc
}

\begin{abstract}
We analyzed 353 Staphylococcus aureus bloodstream isolates from 2004 to 2009 to identify dominant genotypes, changes over time, and associations between genotype, phenotype, and clinical parameters. The isolates were genotyped with regard to spa type and presence of Panton-Valentine leukocidin and toxic shock syndrome toxin 1-encoding genes. A high level of genetic diversity was detected. All but three isolates were methicillin sensitive. Interestingly, spa clonal complex 021 showed a weak association with higher all-cause hospital mortality.
\end{abstract}

$S_{s e d}^{\text {and }}$ taphylococcus aureus is a leading cause of bacteremia often associated with metastatic infections and high levels of morbidity and mortality. In Europe, the occurrence of $S$. aureus bacteremia (SAB) caused by methicillin-sensitive S. aureus (MSSA) is increasing while that of SAB caused by methicillin-resistant $S$. aureus (MRSA) is decreasing (4). It is therefore important to demonstrate whether SAB due to MSSA is limited to certain bacterial clones or clusters of strains and whether they differ geographically.

The aims were to investigate (i) dominant $S$. aureus genotypes causing bacteremia, (ii) frequency shifts of genotypes over time, (iii) associations between genotypes and well-defined clinical subgroups of SAB patients, and (iv) associations between genotypes and resistance to antibiotics.

All S. aureus isolates $(n=364)$ collected from blood cultures in routine diagnostics from July 2004 through December 2009 at Akershus University Hospital, Norway, were obtained. The hospital is an acute somatic hospital with a catchment area of 360,000 inhabitants and more than 40,000 hospitalizations yearly in the selected period. The isolate from the first $S$. aureus-positive blood culture from each patient was included, unless consecutive blood cultures showed a different genotype; in that case (which occurred with four patients), both isolates were included, as they were regarded as unrelated infections. For one patient, only the second consecutive isolate was typeable by spa analysis. Five patients were excluded due to missing isolates, and six patients had untypeable spa genes, leaving a total of 353 isolates for further analysis.

Demographic and clinical data were extracted and reviewed retrospectively from patient charts. Hospital-acquired (HA) and community-acquired (CA) SAB were defined by a positive blood culture for $S$. aureus obtained more and less, respectively, than 48 $\mathrm{h}$ after hospital admission. Endocarditis was defined according to the modified Duke criteria (6). SAB associated with intravascular devices included both short- and long-term arterial and venous catheters. Deep-seated abscesses included all well-defined abscesses in musculature and inner organs, while subcutaneous abscesses were not recorded. Mortality was registered as all-cause in hospital.

spa typing was performed as described previously (1). Assignment of spa type and Based Upon Repeat Pattern (BURP) analysis for assignment of spa clonal complexes (CCs) were performed using Ridom StaphType v.2.0.3 (http://www.ridom.de). The BURP parameters were set as described by Mellman and collaborators $(8)$.

Detection of Panton-Valentine leukocidin (PVL) genes (lukS/ $F-P V)$ on isolates from 2006 to 2007 and tst on all isolates was performed using real-time PCR (RT-PCR) and power SYBR green master mix according to the manufacturer's instructions (Applied Biosystems, Foster City, CA) (2, 3). PVL analysis of the remaining isolates was performed according to an in-house RT-PCR protocol with a minor groove binder (MGB) probe (6-carboxyfluorescein-C AGAATTTATTGGTGTCCTATC), a forward primer published previously (3), and a reverse primer (TTTTGCAGCGTTTTGTT TTCG) according to the manufacturer's instructions for FAST application (Applied Biosystems).

Antibiotic susceptibility was performed by disk diffusion analysis (Bio-Rad, France) according to guidelines and breakpoints of the Norwegian Working Group of Antibiotics (http://www .antibiotikaresistens.no). The antibiotics tested were erythromycin, fusidic acid, gentamicin, oxacillin, penicillin, tetracycline, and trimethoprim-sulfamethoxazole. Sensitive and intermediate isolates were combined in statistical analyses.

Statistical analyses were conducted using SPSS version 15 (SPSS Inc., Chicago, IL). Fisher's exact test was performed for comparison analysis and linear regression for time trend analyses using 6 months as the time interval. A significance level of 0.05 was chosen.

The median age of patients was 70 years (range, 0 to 97 years), and $56 \%$ were men. The clinical subgroups are listed in Table 1. The isolates were assigned to 168 different spa types and 13 spa CCs (Fig. 1 and Table 1), with no significant differences between

\footnotetext{
Received 23 May 2012 Returned for modification 20 June 2012

Accepted 5 July 2012

Published ahead of print 11 July 2012

Address correspondence to Hege Vangstein Aamot,

hege.vangstein.aamot@ahus.no.

Copyright @ 2012, American Society for Microbiology. All Rights Reserved.

doi:10.1128/JCM.01352-12
} 
TABLE 1 spa type, spa clonal complexes, PVL and tst status of 353 S. aureus bloodstream isolates in relationship with patients' clinical characteristics $^{g}$

\begin{tabular}{|c|c|c|c|c|c|c|c|c|}
\hline \multirow[b]{2}{*}{$\begin{array}{l}\text { spa type, spa CC, } \\
\text { PVL, or } t s t\end{array}$} & \multirow[b]{2}{*}{$\begin{array}{l}\text { No. }(\%) \text { of } \\
\text { isolates }\end{array}$} & \multicolumn{7}{|l|}{ No. (\%) with } \\
\hline & & CA infection & HA infection & Endocarditis & $\begin{array}{l}\text { Deep-seated } \\
\text { abscess }^{a}\end{array}$ & Intravascular device & $\begin{array}{l}\text { Osteomyelitis/ } \\
\text { arthritis }^{a}\end{array}$ & $\begin{array}{l}\text { All-cause hospital } \\
\text { mortality }^{a}\end{array}$ \\
\hline \multicolumn{9}{|l|}{ spa type } \\
\hline t084 & $46(13.0 \%)$ & $22(47.8 \%)$ & $24(52.2 \%)$ & $3(6.5 \%)$ & $6(13.4 \%)$ & $15(32.6 \%)^{b}$ & $6(17.6 \%)$ & $14(16.5 \%)$ \\
\hline t002 & $18(5.1 \%)$ & $11(61.1 \%)$ & $7(38.9 \%)$ & $2(11.1 \%)$ & 0 & $3(16.7 \%)^{b}$ & $2(5.9 \%)$ & $5(5.9 \%)$ \\
\hline t065 & $15(4.2 \%)$ & $11(73.3 \%)$ & $4(26.7 \%)$ & $2(13.3 \%)$ & $2(13.3 \%)$ & $0^{b}$ & 0 & $3(3.5 \%)$ \\
\hline $\mathrm{t} 012$ & $14(4.0 \%)$ & $7(50.0 \%)$ & $7(50.0 \%)$ & $1(7.1 \%)$ & 0 & $4(28.6 \%)^{b}$ & $2(5.9 \%)$ & $6(7.1 \%)$ \\
\hline Other spa types ${ }^{c}$ & $260(73.7 \%)$ & $141(54.2 \%)$ & $119(45.8 \%)$ & $13(5.0 \%)$ & $27(10.4 \%)$ & $62(23.8 \%)^{b}$ & $24(70.6 \%)$ & $57(67.1 \%)$ \\
\hline Total & 353 & $192(54.4 \%)$ & $161(45.6 \%)$ & $21(5.9 \%)$ & $35(9.9 \%)$ & $84(23.8 \%)$ & $34(9.6 \%)$ & $85(24.1 \%)$ \\
\hline \multicolumn{9}{|l|}{$\mathrm{CC}$} \\
\hline spa CC084 & $69(20.7 \%)$ & $34(49.3 \%)$ & $35(50.7 \%)$ & $5(7.2 \%)$ & $8(11.6 \%)$ & $19(27.5 \%)$ & $8(11.6 \%)$ & $19(27.5 \%)^{d}$ \\
\hline spa CC021 & $55(16.5 \%)$ & $31(56.4 \%)$ & $24(43.6 \%)$ & $2(3.6 \%)$ & $5(9.1 \%)$ & $13(23.6 \%)$ & $6(10.9 \%)$ & $20(36.4 \%)^{d}$ \\
\hline spa CC133 & $32(9.6 \%)$ & $21(65.6 \%)$ & $11(34.4 \%)$ & $1(3.1 \%)$ & $4(12.5 \%)$ & $6(18.8 \%)$ & $6(18.8 \%)$ & $4(12.5 \%)^{d}$ \\
\hline spa CC065 & $30(9.0 \%)$ & $19(63.3 \%)$ & $11(36.7 \%)$ & $3(10.0 \%)$ & $3(10.0 \%)$ & $3(10.0 \%)$ & $1(3.3 \%)$ & $6(20.0 \%)^{d}$ \\
\hline spa CC002 & $22(6.6 \%)$ & $14(63.6 \%)$ & $8(36.4 \%)$ & $3(13.6 \%)$ & $1(4.5 \%)$ & $3(13.6 \%)$ & $2(9.1 \%)$ & $6(27.3 \%)^{d}$ \\
\hline spa C304/024 & $22(6.6 \%)$ & $12(54.2 \%)$ & $10(45.5 \%)$ & $1(4.5 \%)$ & $3(13.6 \%)$ & $6(27.3 \%)$ & $1(4.5 \%)$ & $3(13.6 \%)^{d}$ \\
\hline spa CC359 & $20(6.0 \%)$ & $11(55.0 \%)$ & $9(45.0 \%)$ & $2(10.0 \%)$ & $4(20.0 \%)$ & $4(20.0 \%)$ & 0 & $6(30.0 \%)^{d}$ \\
\hline Other spa CCs $s^{e}$ & $83(24.9 \%)$ & $41(49.4 \%)$ & $42(50.6 \%)$ & $4(4.8 \%)$ & $6(7.3 \%)$ & $26(31.3 \%)$ & $9(11.0 \%)$ & $15(18.3 \%)^{d}$ \\
\hline Total & 333 & $183(55.0 \%)$ & $150(45.0 \%)$ & $21(6.3 \%)$ & $34(10.2 \%)$ & $80(24.0 \%)$ & $33(9.9 \%)$ & $79(23.7 \%)$ \\
\hline PVL & $11(3.1 \%)^{f}$ & $10(90.9 \%)^{f}$ & $1(9.1 \%)^{f}$ & $1(9.1 \%)$ & $3(27.3 \%)$ & $2(18.2 \%)$ & $1(9.1 \%)$ & $2(18.2 \%)$ \\
\hline tst & $54(15.3 \%)$ & $28(51.9 \%)$ & $26(48.1 \%)$ & $1(1.9 \%)$ & $4(7.4 \%)$ & $16(29.6 \%)$ & $6(11.1 \%)$ & $16(29.6 \%)$ \\
\hline
\end{tabular}

${ }^{a}$ One sample was excluded due to lack of information.

${ }^{b} P=0.027$ for $s p a$ type 065 versus other spa types: $0 \%$ versus $23.8 \%$.

${ }^{c}$ Consists of 164 unique spa types.

${ }^{d} P=0.023$ for spa CC021 versus other spa CCs: $36.4 \%$ versus $24.9 \%$.

${ }^{e} 20$ isolates (5.7\%) excluded from BURP analysis, 5 CCs had no founder, 16 isolates were singletons.

${ }^{f} P=0.014$ for PVL in CA infection versus HA infection: $90.9 \%$ versus $9.1 \%$.

$g$ Only statistically significant $P$ values are presented.

HA and CA infections. Their frequencies coincide with those in other studies from Norway on nasal S. aureus carriers (11-13), suggesting that there are no locally or geographically dominating spa types or clusters associated with invasive MSSA infections. The degree of accordance between carrier and invasive $S$. aureus spa types may be partly explained by the high frequency of endogenous infections (15).

The PVL toxin has generally been associated with localized skin and soft tissue CA infections and necrotizing pneumonia $(5,7)$, but its significance in SAB is less known. As only $3.1 \%$ of the isolates were positive for the PVL genes, the role of PVL as a virulence factor of invasive infections remains unproven. Accordingly, PVL showed the only significant difference between CA and HA infections, where 10 of 11 PVL-positive isolates were CA infections (Table 1).

The frequency of isolates containing the tst gene (15.3\%) correlates well with previous findings on $\mathrm{SAB}$ and asymptomatic $S$. aureus carriers $(9,14)$.

No shifts in frequency of spa type, spa CC, PVL, tst, or antibiotic resistance over time during the 5.5-year study period could be established, suggesting that the common S. aureus population in the hospital's catchment area is relatively stable over time.

The correlations of genotypes and clinical subgroups are presented in Table 1. Of specific interest, patients with spa CC021 isolates had a higher all-cause hospital mortality rate than patients with other spa CCs (36.4\% versus $21.3 \%, P=0.023)$. To our knowledge this has not been reported previously. Because other factors likely to affect mortality, such as comorbidity and disease severity, were not accounted for, it is not known if spa CC021 represents an independent predictor. However, the association is weak and needs to be demonstrated in future prospective studies. As the methods utilized have relatively low resolution, whole-genome analysis could provide additional insight into the possible increased virulence of spa CC021 strains and the population genetic structure overall. Also, virulence determinants may differ among and between genotypes, so genotyping alone may not be predictive of disease associations.

It is noteworthy that a reported association between MSSA endocarditis and multilocus sequencing type (MLST) CC30 (10) could not be confirmed in this study, as only 2 of 21 patients with endocarditis had a spa type corresponding to MLST CC30.

The frequencies of antibiotic resistance were low, with the exception of penicillin, to which 262 isolates (74.2\%) were resistant. Only three isolates were resistant to methicillin (MRSA) (0.8\%), and less than $6 \%$ of the isolates were resistant to the other antibiotics. The low MRSA frequency was to be expected due to the low prevalence of MRSA bloodstream infections reported in Norway. The overall low prevalence of antibiotic resistance suggests that in our setting SAB isolates do not represent a large reservoir of antibiotic resistance genes.

Our study confirms the large genetic diversity of MSSA bacte- 

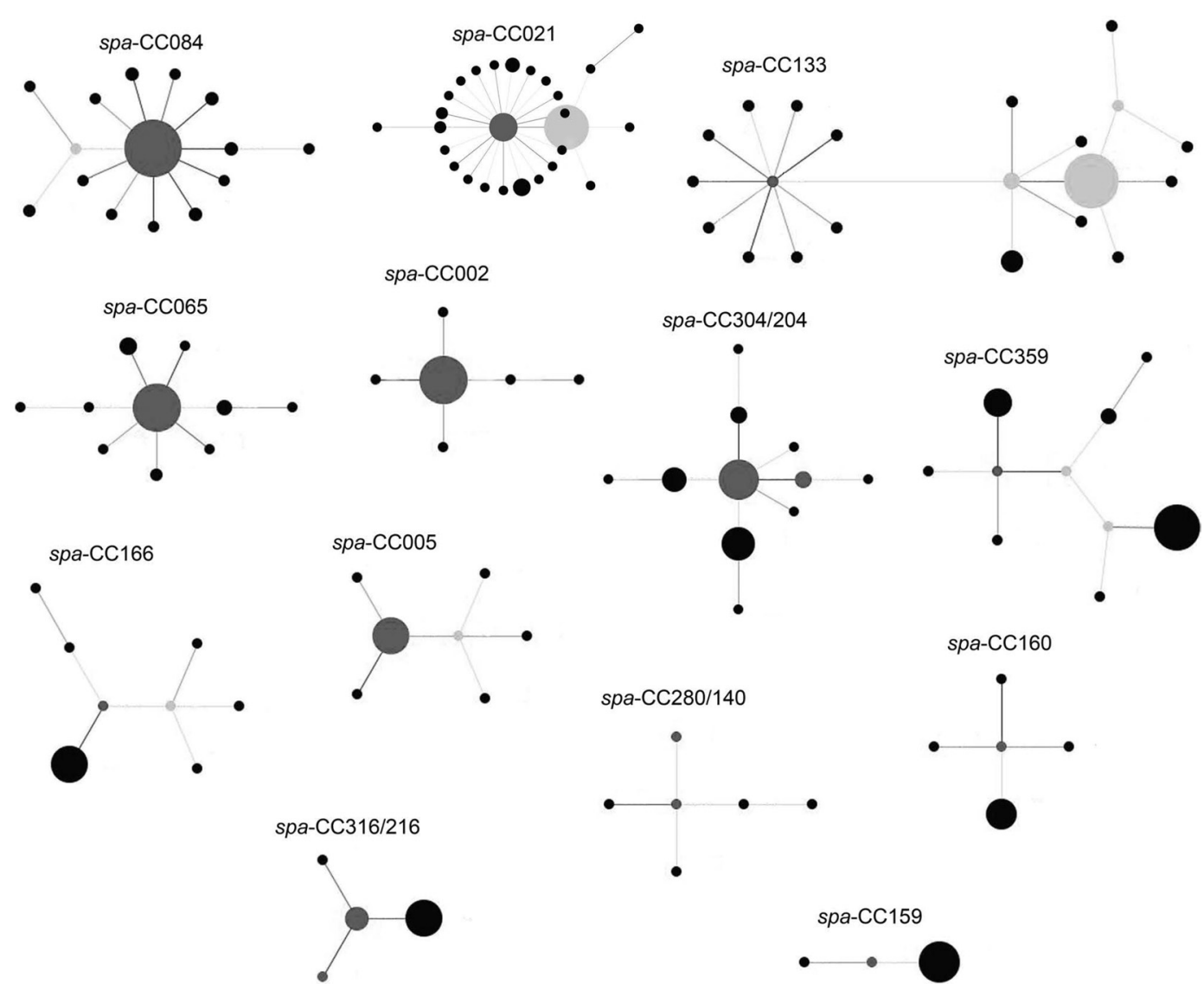

\section{spa-CC159}

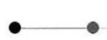

FIG 1 spa clonal complexes of the 353 S. aureus bacteremia isolates after Based Upon Repeat Pattern (BURP) analysis show genetic heterogeneity. spa CCs without founder, singletons, and 20 isolates not fulfilling the BURP analysis criteria (8) are not included in the figure. Clusters of linked isolates correspond to spa CCs. The spa type with the highest founder score is defined as the founder of the cluster and is marked in dark gray. Light gray indicates subfounders, which are the spa types with the second highest founder score. If two spa types have an equally high founder score, both are colored dark gray. Only the spa CCs are labeled. The size of the circles indicates the number of isolates that were assigned to that spa type. Note that the spacing between linked and unlinked spa types and spa CCs does not provide information about the genetic distance between them. The different clonal complexes consist of the following spa types. spa CC 021: $\mathrm{t} 012, \mathrm{t} 017, \mathrm{t} 018, \mathrm{t} 019, \mathrm{t} 021, \mathrm{t} 037, \mathrm{t} 046, \mathrm{t} 122, \mathrm{t} 238, \mathrm{t} 318, \mathrm{t} 342, \mathrm{t} 486, \mathrm{t} 582, \mathrm{t} 584, \mathrm{t} 726, \mathrm{t} 840, \mathrm{t} 869, \mathrm{t} 1298, \mathrm{t} 1347, \mathrm{t} 1414$, t2303, t3222, t3611, t4033, t6360, t6361, t6362; spa CC 133: t015, t050, t116, t133, t230, t295, t331, t722, t728, t908, t1078, t1081, t1510, t1996, t2519, $\mathrm{t} 3537, \mathrm{t} 4604, \mathrm{t} 5025, \mathrm{t} 6259, \mathrm{t} 6368, \mathrm{t} 6414 ;$ spa CC 084: $\mathrm{t} 084, \mathrm{t} 085, \mathrm{t} 120, \mathrm{t} 279, \mathrm{t} 328, \mathrm{t} 346, \mathrm{t} 491, \mathrm{t} 774, \mathrm{t} 853, \mathrm{t} 1034, \mathrm{t} 1116, \mathrm{t} 1492, \mathrm{t} 1877, \mathrm{t} 3603, \mathrm{t} 4714 ;$ spa CC 304/024: t008, t024, t190, t304, t594, t648, t701, t2842, t5160, t6269, t6363; spa CC 065: t004, t040, t065, t123, t130, t330, t370, t715, t880, t6413; spa CC 359: t044, t127, t177, t189, t224, t267, t359, t1381, t6367, t6369; spa CC 166: t089, t136, t153, t166, t414, t2103, t3631, t5741; spa CC 005: t005, t223, t309, t474, t490, t1139, t6265; spa CC 002: t002, t010, t045, t067, t214, t1265; spa CC 280/140: t140, t167, t258, t280, t1541, t3188; spa CC 160: t156, t160, t213, t771, t1137; spa CC 316/216: t172, t216, t316, t471; spa CC 159: t159, t171, t284.

remias and supports the perception that almost any S. aureus strain may cause invasive infections.

\section{ACKNOWLEDGMENTS}

We thank the Department of Microbiology and Infection Control at Akershus University Hospital, Norway, for diagnostic analyses of the S. aureus isolates and Anwar Kiyamow, Lydia Nyland Lineikro, and Janne Sølvernes for their technical assistance with genotyping analyses.

\section{REFERENCES}

1. Fossum AE, Bukholm G. 2006. Increased incidence of methicillinresistant Staphylococcus aureus ST80, novel ST125 and SCCmecIV in the south-eastern part of Norway during a 12-year period. Clin. Microbiol. Infect. 12:627-633.

2. Fossum Moen AE, Saltyte BJ, Alm-Kristiansen K, Bukholm G. 2009. Exotoxin-encoding gene content in community-acquired and hospitalacquired methicillin-resistant Staphylococcus aureus. Clin. Microbiol. Infect. 15:1139-1145.

3. Francois P, et al. 2004. A novel multiplex real-time PCR assay for rapid typing of major staphylococcal cassette chromosome mec elements. J. Clin. Microbiol. 42:3309-3312.

4. Gagliotti C, et al. 2011. Escherichia coli and Staphylococcus aureus: bad news and good news from the European Antimicrobial Resistance Surveillance Network (EARS-Net, formerly EARSS), 2002 to 2009. Euro Surveill. 16(11):pii=19819. http://www.eurosurveillance.org/ViewArticle.aspx ?ArticleId $=19819$.

5. Gillet Y, et al. 2002. Association between Staphylococcus aureus strains carrying gene for Panton-Valentine leukocidin and highly lethal necrotising pneumonia in young immunocompetent patients. Lancet 359:753759.

6. Li JS, et al. 2000. Proposed modifications to the Duke criteria for the diagnosis of infective endocarditis. Clin. Infect. Dis. 30:633-638.

7. Lina G, et al. 1999. Involvement of Panton-Valentine leukocidinproducing Staphylococcus aureus in primary skin infections and pneumonia. Clin. Infect. Dis. 29:1128-1132.

8. Mellmann A, et al. 2007. Based Upon Repeat Pattern (BURP): an algorithm to characterize the long-term evolution of Staphylococcus aureus populations based on spa polymorphisms. BMC Microbiol. 7:98.

9. Monecke S, Luedicke C, Slickers P, Ehricht R. 2009. Molecular epide- 
miology of Staphylococcus aureus in asymptomatic carriers. Eur. J. Clin. Microbiol. Infect. Dis. 28:1159-1165.

10. Nienaber JJ, et al. 2011. Methicillin-susceptible Staphylococcus aureus endocarditis isolates are associated with clonal complex 30 genotype and a distinct repertoire of enterotoxins and adhesins. J. Infect. Dis. 204:704-713.

11. Sangvik M, et al. 2011. Age- and gender-associated Staphylococcus aureus spa types found among nasal carriers in a general population: the Tromso Staph and Skin Study. J. Clin. Microbiol. 49:4213-4218.

12. Skramm I, Moen AE, Bukholm G. 2011. Nasal carriage of Staphylococcus aureus: frequency and molecular diversity in a randomly sampled Norwegian community population. APMIS 119:522-528.
13. Skramm I, Moen A, Alm-Kristiansen K, Bukholm G. 2007. Nasal carriage of Staphylococcus aureus: which sequence types do orthopedic surgical healthcare workers carry? Infect. Control Hosp. Epidemiol. 28:737739.

14. van der Mee-Marquet N, et al. 2009. Variable-number tandem repeat analysis and multilocus sequence typing data confirm the epidemiological changes observed with Staphylococcus aureus strains isolated from bloodstream infections. J. Clin. Microbiol. 47:2863-2871.

15. Wertheim HF, et al. 2004. Risk and outcome of nosocomial Staphylococcus aureus bacteraemia in nasal carriers versus non-carriers. Lancet 364: 703-705. 